\title{
Désaturer et naturaliser l'esprit. Deux usages du pragmatisme
}

Quelques notes sur le livre de Pierre Steiner

\section{Roberta Dreon}

\section{(2) OpenEdition}

\section{Journals}

Édition électronique

URL : http://journals.openedition.org/ejpap/1952

DOI : 10.4000/ejpap.1952

ISSN : 2036-4091

\section{Éditeur}

Associazione Pragma

\section{Référence électronique}

Roberta Dreon, « Désaturer et naturaliser l'esprit. Deux usages du pragmatisme », European Journal of Pragmatism and American Philosophy [En ligne], XII-1 | 2020, mis en ligne le 06 juin 2020, consulté le 26 juin 2020. URL : http://journals.openedition.org/ejpap/1952 ; DOI : https://doi.org/10.4000/ejpap.1952

Ce document a été généré automatiquement le 26 juin 2020.

\section{(c) $($ ) $(9)$}

Author retains copyright and grants the European Journal of Pragmatism and American Philosophy right of first publication with the work simultaneously licensed under a Creative Commons AttributionNonCommercial-NoDerivatives 4.0 International License. 


\title{
Désaturer et naturaliser l'esprit. Deux usages du pragmatisme
}

\author{
Quelques notes sur le livre de Pierre Steiner
}

\author{
Roberta Dreon
}

$1 \quad$ Le livre de Pierre Steiner, Désaturer l'esprit. Usages du pragmatisme, publié l'année dernière par la maison d'édition Questions Théoriques, se démarque par la richesse de ses discussions des auteurs et des thèmes au cœur du débat philosophique contemporain. Les analyses, jamais superficielles ni surchargées, sont toujours pertinentes et approfondies. Les quatre philosophes qui font l'objet des discussions les plus intenses constituent différents modèles de compréhension du pragmatisme: on passe du néo-pragmatisme linguistique de Richard Rorty au pragmatisme normatif de Robert Brandom, puis du pragmatisme sui generis de Ludwig Wittgenstein au pragmatisme naturaliste classique et expérimental de John Dewey. Ces discussions sont accompagnées de références (explicites et implicites) à de nombreux autres auteurs, comme Joseph Margolis et Larry Hickman, ainsi que le regretté Jean-Pierre Cometti, auquel le livre est dédié. Il y a de nombreux renvois aux débats en philosophie de l'esprit (sur les neurosciences et les positions énactivistes, le dualisme corps-esprit, la contingence, l'émergence, les solutions éliminativistes et monistes), en philosophie des sciences (sur le scientisme et ses implications politiques) et en philosophie de la technique (sur la neutralité de la technique et la démocratie). De plus, Steiner reprend la discussion sur le respect d'une règle et la normativité, ainsi que la discussion sur l'expressivité humaine et la tendance à hypostasier les phases et les modes d'expérience. Tout cela est maîtrisé avec beaucoup de compétence et d'adresse.

2 Cette richesse de sources, de thèmes et de positions ne constitue nullement un obstacle à la poursuite lucide d'un parcours bien précis, tracé par Steiner, qui utilise toutes les ressources disponibles dans le but explicite du titre du livre: effectuer une désaturation de l'esprit (mind), proposer une interprétation déflationniste de son concept, éviter toute forme d'hypostase et repenser l'esprit en termes de vocabulaire, d'option lexicale enracinée dans un contexte culturel spécifique (avec Rorty), ou bien en termes d'engagements normatifs inhérents aux pratiques linguistiques (avec Brandom), ou 
encore dans les termes subtils de l'expressivité de Wittgenstein, qui renonce au dualisme entre intériorité et comportement, ou enfin, avec Dewey, sous une forme adverbialiste, comme signification (meaning) et qualité qui concerne les transactions des agents humains avec le monde. Tout cela en résistant ascétiquement, comme le Christ dans le désert, à toute tentation métaphysique, même à celle que Steiner croit encore trouver dans l'énactivisme, selon lequel les événements mentaux sont assimilés à des processus qui seraient déjà là (non plus dans l'esprit, mais étendus dans le corps et le milieu environnant), qu'il s'agirait toujours d'expliquer.

En résumé, on pourrait dire que le style et le goût qui caractérisent ce livre sont sans doute européens et français, mais que la qualité et la clarté argumentative du texte répondent pleinement aux plus hauts standards de la littérature philosophique anglophone.

4 Afin de maintenir un certain ordre dans mon exposé, je m'attarderai sur les quatre étapes du parcours de "désaturation" de l'esprit proposé par Steiner, en insérant de temps en temps quelques considérations sur des aspects qui pourraient être davantage précisés ou explorés. ${ }^{1}$ Enfin, dans un dernier temps, je présenterai une réflexion critique sur le parcours dans son ensemble, en soutenant qu'en épousant l'approche de l'esprit proposée par Dewey, le discours ne se limite pas à une proposition déflationniste, soucieuse d'ôter toute approche ontologique et épistémologique qui alourdirait la question ; le discours deweyien implique également des engagements positifs en faveur d'une forme de naturalisme non réductionniste, culturel et émergent. Le parcours habilement tracé par l'auteur ne me semble pas pouvoir se dérouler de manière linéaire et sans tension, entre l'"éliminativisme thérapeutique" de Rorty (Steiner 2019: 72) et la conception adverbialiste de l'esprit de Dewey, qui implique une approche naturaliste non réductionniste comme pars construens. Les deux stratégies ne sont pas nécessairement incompatibles. Par contre, contrairement à Rorty et à ses critiques des restes métaphysiques de Dewey, je crois personnellement (et Steiner serait probablement d'accord avec moi) que les deux stratégies peuvent être maintenues ensemble en recourant à une conception de la nature humaine qui n'est pas aprioriste, mais qui est liée et ouverte aux contingences de son évolution. Mais il faut néanmoins reconnaître qu'il s'agit de deux usages différents et éventuellement complémentaires du pragmatisme.

\section{I.}

5 Dans son projet d'offrir une "approche post-ontologique de l'esprit et de la cognition" (12), la confrontation avec Rorty joue un rôle décisif. Elle guide tout le parcours de Steiner, même au-delà du premier chapitre. À la lumière des enquêtes sur le problème corps-esprit menées avant la publication de Philosophy and The Mirror of Nature en 1979, Steiner note que la stratégie de Rorty consiste à déplacer la question du plan de l'ontologie à celui des vocabulaires. Pour Rorty, l'esprit ne préexiste pas à l'ensemble des pratiques linguistiques avec lesquelles nous en parlons, mais il existe dans les conditions prescrites par nos coutumes et pratiques linguistiques. De plus, aucun vocabulaire - ni le vocabulaire psycho-philosophique du mental, ni le vocabulaire scientifique des processus neuronaux - n'a de relation privilégiée avec la réalité que, selon Rorty, les hommes ne touchent qu'à l'intérieur d'une langue. L'imprécision des limites du mental n'est donc pas une indication de sa profondeur ou de son inefficacité, 
mais indique plutôt la diversité des vocabulaires dans lesquels le mot "esprit" est nécessaire. Pour résoudre le problème corps-esprit, il faut donc renoncer à la réification indue de deux types de vocabulaires différents, c'est-à-dire de deux types de pratiques sociales différentes. La stratégie de retranscription de la question ontologique en termes de vocabulaire est ensuite complétée par l'antireprésentationnalisme radical de Rorty: les termes d'un vocabulaire ne sont pas vrais parce qu'ils désignent quelque chose dans le monde extra-linguistique, mais plutôt parce qu'ils permettent de faire des choses différentes, de poursuivre des fins alternatives. L'irréductibilité d'un vocabulaire à un autre n'est pas due à l'accès présumé à la réalité elle-même, mais aux différentes conséquences des activités linguistiques dans lesquelles se réalisent les relations de l'homme au monde.

Dans la manière dont Steiner reconstruit la position de Rorty, certains éléments - qui mériteraient d'être davantage valorisés - montrent que son analyse de la question ne se réduit pas à une forme banalisée de constructivisme et de relativisme linguistique. D'une part, la notion de vocabulaire de Rorty est tout sauf purement linguistique, incluant plutôt "un ensemble historique de significations, de propositions, de descriptions, de croyances, de métaphores et de valeurs, intimement lié à ce que nous faisons et voulons faire dans un contexte donné" (56). En d'autres termes, le langage n'est pas considéré comme une entité autonome distincte du monde, mais il est compris comme une pluralité contingente de pratiques avec lesquelles les humains font des expériences effectives des contextes dans lesquels ils vivent et agissent - bref, ce n'est pas le monde tout court qui est définitivement perdu, mais l'hypothèse du réalisme dogmatique d'un monde totalement déterminé, indépendant des pratiques humaines, tout comme l'hypothèse du langage comme système essentiellement autonome, indifférent aux conséquences existentielles de ses applications. D'autre part, précisément, les pratiques linguistiques ne sont pas détachées de l'existence réelle; au contraire, leur valeur de vérité est mesurée par leur capacité à poursuivre certains buts (Calcaterra 2019: 23 sq.). De ce point de vue, il convient de souligner qu'une approche cohérente de la position de Rorty devrait conduire à une atténuation de l'opposition simpliste entre le langage et l'expérience (alimentée par certaines formulations hâtives de Rorty), comme élément clé du passage du pragmatisme classique au pragmatisme linguistique. ${ }^{2}$

\section{II.}

7 Pour nous conduire à une conception déflationniste de l'esprit, Steiner mobilise dans un second temps le pragmatisme de Robert Brandom, en en faisant un usage double et original. Tout d'abord, bien qu'il critique certaines ambiguïtés de Brandom liées à ses concessions représentationnalistes, Steiner estime que la proposition du philosophe américain peut être utilisée efficacement pour critiquer la conception internaliste de la pensée, c'est-à-dire pour soutenir que la pensée humaine consiste en une série de pratiques normatives entre locuteurs, assumant ainsi une conception constructiviste des épisodes mentaux. De plus, l'exploration de la thèse de Brandom sur la structure normative des pratiques linguistiques humaines lui permet d'aborder de manière alternative le problème du rôle à attribuer aux événements cérébraux dans la réalisation du comportement mental. Le modèle à éliminer est la conception standard, que Steiner nomme "internalisme véhiculaire" (Steiner 2019: 103), selon laquelle les 
événements neuronaux sont des véhicules ou des supports pour la réalisation d'“épisodes mentaux conceptuels" (102), ce sont des entités matérielles ou des processus sans propriétés sémantiques qui véhiculent un contenu sémantique - une pensée, un sens, un concept. L'inférentialisme de Brandom, en revanche, conduit à une dislocation de la signification dans les pratiques sociales où elle se constitue: la signification d'un énoncé ne se fonde pas sur des entités mentales déjà données, mais elle se constitue au cours de l'échange, comme règle de la relation entre les interlocuteurs en rapport avec les actions qu'ils peuvent entreprendre et les objets sur lesquels les pratiques s'appuient. Dans l'“inférentialisme large" de Brandom (115), Steiner souligne la dimension holistique - je dirais réaliste, en revendiquant un usage non dogmatique du terme - qui lie les pratiques linguistiques aux circonstances d'application d'un concept et aux conséquences de son emploi: dans les deux cas, il ne s'agit pas simplement d'activités linguistiques, mais d'activités impliquant des engagements effectifs. En effet, en parlant, chacun prend plus ou moins consciemment des engagements avec l'interlocuteur, notamment en ce qui concerne la prétention de vérité de ses propres propositions, chacun envisage une certaine compatibilité et exclut des incompatibilités avec les usages de l'autre locuteur, chacun se sent autorisé à juger d'une certaine manière sur la base d'inférences spécifiques. L'influence wittgensteinienne est évidente dans la mise en valeur de la dimension normative des pratiques, qui devient prépondérante: comprendre une signification, ce n'est pas appréhender un contenu conceptuel déjà donné dans l'esprit, mais c'est maîtriser l'utilisation d'un concept, sa structure inférentielle et normative, c'est assumer les droits et les engagements inhérents aux pratiques linguistiques humaines.

Par une démarche originale, Steiner reconfigure ici le rôle du cerveau dans la production d"'événements mentaux conceptuels": les processus cérébraux ne sont pas les véhicules ou les supports de contenus sémantiques déjà déterminés au niveau mental avant les pratiques de profération linguistique - l'inférentialisme large de Brandom conduit à démystifier une telle conception de la signification - et, à ce niveau, il n'y a tout simplement pas besoin de véhicule, puisqu'il n'y a pas de contenu déjà prêt à être transporté. Pour Steiner, le rôle du cerveau dans le développement d'événements mentaux conceptuels est indirect et devrait être formulé différemment, au niveau du comportement inférentiel avec lequel les intervenants considèrent les circonstances et les conséquences réelles liées à l'utilisation de certains lexiques. Fort d'une bonne dose de provocation, Steiner conclut que "[...] en considérant son rôle dans la production et l'existence d'EMC [Épisodes Mentaux Conceptuels], le cerveau joue le même rôle qu'un muscle: il cause et contraint la capacité à déployer un comportement inférentiel" (127). Les questions de savoir quel type de causalité est en jeu, et comment elle est réalisée, devraient être posées à la lumière des ressources les plus avancées fournies par les théories post-cognitives de l'esprit: le cerveau a-t-il une suprématie sur le corps dans l'organisme humain? Sa capacité causale est-elle efficace et unidirectionnelle, ainsi qu'indépendante de son lien avec un environnement donné? Le traitement de ces questions implique un approfondissement des conceptions de l'esprit embodied, embedded, extended et enacted que Steiner, grand connaisseur de ce débat (voir Steiner 2014 et Steiner 2017), pourrait davantage explorer. 
III.

9 Dans la troisième phase du parcours tracé dans ce livre, Steiner se consacre justement à la discussion de la contribution des sciences post-cognitives à une conception déontologisée de l'esprit, mais il préfère se concentrer sur une critique de l'énactivisme avec des arguments dérivés de Wittgenstein. Le programme le plus radical de l'énactivisme soutient une forme d'externalisme cognitif, qui ne nie pas l'existence des processus cérébraux, mais qui considère que le corps et l'environnement ont également un rôle constitutif dans la cognition, comprise comme une action incorporée dans un environnement et en continuité avec les phénomènes vitaux. Néanmoins, Steiner estime que, en dépit de cette importante convergence, l'énactivisme, même dans ses formes anti-représentationnalistes et anti-computationnelles, maintient deux hypothèses problématiques. Tout d'abord, ce que l'auteur appelle "le mythe des processus mentaux": l'assimilation de tous les événements mentaux à des processus mentaux est trompeuse, car elle suggère qu'ils sont déjà là, quelque part, et qu'il faut les expliquer et les désigner. Avec Wittgenstein, Steiner répond que, lorsqu'on traite des phénomènes mentaux et des crampes philosophiques qui en résultent, il faut changer d'approche: au lieu de se focaliser sur les différentes formes d'expérience - sur l'attitude phénoménologique et sur l'attitude scientifique à la troisième personne - il faut observer les différentes occurrences des mots et des concepts utilisés dans différents contextes. Si dans certains cas la caractérisation du mental en termes de processus est acceptable, l'extension de cette identification à toutes les occurrences du mental est trompeuse. Le fait est que (il s'agit ici de la deuxième critique que Steiner oppose à l'énactivisme) les concepts psychologiques ne figurent pas principalement dans le discours ordinaire en tant que concepts théoriques, à l'égard desquels nous ressentons l'obligation de chercher une explication en termes ontologiques et épistémologiques (Perissinotto 2018: 218-9). Ils sont également présents dans les conversations quotidiennes afin de nous permettre de comprendre les autres, de comprendre leurs actions ou leurs dispositions à l'action, et aussi de réguler notre conduite par rapport à celle des autres. D'après la lecture que Steiner fait de Wittgenstein, un visage exprime une émotion et un geste exprime une forme d'action, non pas parce que dans l'intériorité humaine quelque chose comme un état mental ou émotionnel préexiste à son extériorisation dans un sourire ou dans une action du corps, mais bien parce que dans l'espace de la vie humaine partagée et dans l'espace de l'action qui a une valeur sociale, une distinction se produit entre ce qui est exprimé et ce qui l'exprime. Une telle distinction a une valeur fonctionnelle en dirigeant la conduite des interlocuteurs vers une discrimination subtile qui permet d'orienter l'action, malgré le haut degré d'imprévisibilité du comportement humain. En d'autres termes, il n'y aurait pas d'être plus profond, mental ou psychique, préalable à son expression, mais la distinction entre les aspects mentaux et la dimension du comportement se ferait à la surface. Il s'agit toutefois d'une surface capable de nuances subtiles, déterminantes dans la régulation réciproque de l'action pacifique ou conflictuelle. 
IV. l'esprit, défendue par Dewey. Dans un passage de Art as Experience (Dewey 1989: 298), dans lequel il traitait des préjugés psychologiques qui minent la théorie de l'art, Dewey proposait de considérer les occurrences du terme "mind" dans les discours ordinaires, en soulignant que dans la plupart des cas "mind" figure comme un verbe - faire attention, prendre soin, se souvenir - plutôt que comme un nom (Dreon 2017: 93). Plus tôt déjà, dans Experience and Nature (Dewey 1981: 126), Dewey suggérait de considérer le mental non pas comme une sorte d'entité, mais en termes adverbiaux, comme une forme particulière d'interaction entre les agents et leur environnement (Steiner 2019: 192). Steiner insiste à juste titre sur les deux aspects de la théorie adverbiale qu'il tire de Dewey. Le premier de ces aspects est que l'adjectif "mental" ou l'adverbe "mentalement" caractérisent avant tout une relation - celle entre l'être humain et son environnement social et culturel; ils concernent la qualité de la relation elle-même, et non l'un des deux pôles (le sujet et l'objet, la noèse et le noème) qu'elle met en corrélation. C'est pourquoi Steiner insiste sur la primauté de la relation dans la philosophie de Dewey, dans laquelle de nombreuses influences hétérogènes se conjuguent - non seulement l'intérêt du pragmatiste américain pour la physique postnewtonienne, mais j'ajouterais également le poids de l'ontologie relationnelle de Peirce (Tiercelin 2019) et de l'empirisme radical de James (Alexander 1987: 72), son appropriation anti-substantialiste et anti-déterministe des thèses de Darwin (Cometti 2016: 62) et, avant cela, l'héritage de la dialectique hégélienne (Good 2006). De ce point de vue, il semble significatif, comme le souligne l'auteur, que la naissance de la science moderne consiste à expulser les qualités secondes de l'objet. Ces dernières n'étant pas soumises à la mathématisation, elles sont attribuées au sujet. Autrement dit, la science moderne montre son incapacité à penser les qualités mentales comme des modes de relations plutôt que comme des entités indépendantes.

Le deuxième aspect que souligne Steiner concerne la spécificité du mode d'interaction mentale. Pour Dewey, le mental est "quasi-synonyme" de signification (Steiner 2019: 198): les interactions des êtres humains sont significatives, c'est-à-dire mentales, parce qu'elles ont lieu dans un milieu social caractérisé par une coopération communicationnelle et linguistique, en vue de la réalisation de buts communs, à savoir la régulation mutuelle des actions des locuteurs. En fait, pour Dewey, la pensée émerge de circonstances sociales caractérisées par des pratiques linguistiques partagées, car la signification exige qu'au moins deux interlocuteurs convergent sur un objet en vue d'un autre, c'est-à-dire qu'ils convergent sur une pratique commune. Quoi qu'il en soit, ce qui reste caché dans le discours de Steiner, c'est que la conception adverbiale de l'esprit de Dewey fonctionne non seulement comme un outil critique ou pars destruens de la compulsion philosophique à substantialiser l'esprit, mais qu'elle implique aussi une certaine conception, naturaliste et émergente, des relations organiquesenvironnementales qu'elle qualifie. En d'autres termes, l'approche de Dewey ne se limite pas à opérer une distinction entre les vocabulaires ou les jeux linguistiques, ni même entre les engagements normatifs, mais elle embrasse une conception des relations que l'on peut dire mentales: les interactions entre les organismes humains et leur environnement naturellement social deviennent mentales, par rapport à d'autres types d'interactions animales qui privilégient la sensibilité et une forme de

European Journal of Pragmatism and American Philosophy, XII-1 | 2020 
signification organique, qui ne se basent pas encore sur l'action différée, mais sur l'impact exercé par certaines circonstances sur la vie de l'organisme en question. ${ }^{3}$ L'émergence contingente mais irréversible de la communication linguistique joue par conséquent un rôle central dans le naturalisme culturel de Dewey, puisqu'elle génère des formes de signification très complexes et nouvelles, c'est-à-dire des formes d'orientation des êtres humains dans l'environnement, à partir de ressources préexistantes dont la réorganisation produit de nouvelles propriétés - des propriétés mentales liées à de nouveaux modes de communication et de signification. Évidemment, Steiner reconnaît que Dewey suppose une continuité fondamentale entre le développement biologique et le développement mental et culturel, et il souligne que le concept d'émergence joue un rôle crucial pour expliquer l'origine des interactions mentales entre les organismes et l'environnement, "dans un cadre réaliste et à partir d'un naturalisme culturaliste" (Steiner 2019: 201). Par contre, je ne suis pas sûre que Steiner soit pleinement conscient de la nouveauté que constitue l'histoire naturelle de l'esprit de Dewey par rapport à la vision exposée jusqu'alors. La déconstruction du problème corps-esprit et sa traduction en termes de vocabulaire et d'engagements normatifs ont sans aucun doute un rôle thérapeutique face à l'inflationnisme métaphysique qui subsiste dans le débat philosophique, et elles semblent s'inscrire dans la conception adverbiale de Dewey, que Steiner aborde à la fin de sa réflexion. Il s'agit cependant de comprendre dans quelle mesure cela est compatible avec l'histoire naturelle de l'esprit tracée par Dewey, qui constitue la pars construens de son approche adverbiale, et qui récupère l'organique, la sensibilité, le qualitatif bien au-delà de ce que Rorty et peut-être Wittgenstein lui-même seraient prêts à concéder. ${ }^{4}$ Il existe à mon avis une tension importante entre les différents usages que nous pouvons et voulons faire du pragmatisme - au-delà des étiquettes de pragmatisme "analytique" et "post-analytique" que, à la fin de son introduction, Steiner considère à juste titre comme stériles.

Steiner n'aborde pas ce point, peut-être en raison des différents chemins qu'il a empruntés tout au long de son parcours pragmatiste (Cometti 2010: 102): Rorty et la question des vocabulaires, qui sont contraints par des pratiques et des conditions spécifiques; Brandom et l'idée que la communication linguistique est d'abord un espace normatif, et pas un espace dans lequel les liens humains sont tissés, maintenus ou démêlés; l'obsession wittgensteinienne pour le grammatical; et une lecture de Dewey à partir de la logique de l'enquête et de sa comparaison avec l'expérimentalisme à l'œuvre dans la physique classique et la physique post-newtonienne.

13 Tout cela est juste mais incomplet, et devrait à mon avis être intégré à la partie plus naturelle du naturalisme culturel que je partage avec Steiner, sans qu'il ait à craindre de se compromettre avec la métaphysique - le fantôme de Rorty qui critique la métaphysique de Dewey continue peut-être à planer (Rorty 1977). Il est certain que nous devons suivre soit les indications de Wittgenstein pour échapper aux crampes philosophiques dues à la façon dont nous utilisons le langage, soit les indications de Dewey pour éviter les erreurs dualistes, l'hypostase excessive, les compulsions à poursuivre la reductio ad unum. Et le naturalisme culturel est certainement un postulat, comme l'affirme Steiner (Steiner 2019: 201). Cependant, contrairement à l'approche du dernier Wittgenstein, le pragmatisme n'est pas seulement une philosophie. Il implique plutôt que nous ne pouvons pas nous débarrasser de toutes les croyances, précisément parce qu'il ne s'agit pas seulement de croyances que $p$ : au contraire, que nous en ayons 
conscience ou pas, nous ne pouvons partir que d'une option spécifique qui fonctionne déjà comme une disposition à agir ainsi qu’à faire de la philosophie.

\section{BIBLIOGRAPHIE}

ALEXANDER Thomas, (1987), John Dewey's Theory of Art, Experience and Nature. The Horizons of Feeling, Albany, State University of New York Press.

CALCATERRA Rosa Maria, (2019), Contingency and Normativity. The Challanges of Richard Rorty, LeidenBoston, Brill Rodopi.

ColombetTI Giovanna, (2014), The Feeling Body. Affective Science Meets the Enactive Mind, CambridgeLondon, The MIT Press.

Cометті Jean-Pierre, (2010), Qu'est-ce le pragmatisme?, Paris, Gallimard.

сометті Jean-Pierre, (2016), La démocratie radicale. Lire John Dewey, Paris, Gallimard.

DEWEY John, (1981), Experience and Nature, Vol. 1 of The Later Works, Carbondale and Edwardsville, Southern Illinois University Press.

DEWEY John, (1989), Art as Experience, Vol. 10 of The Later Works, Carbondale and Edwardsville, Southern Illinois University Press.

DREON Roberta, (2017), Sortir de la tour d'ivoire. L'esthétique inclusive de John Dewey aujourd'hui, trad. J. Orsini, Paris, Questions Théoriques.

GOOD James A., (2006), A Search for Unity in Diversity. The "Permanent Hegelian Deposit" in the Philosophy of John Dewey, Lanham, Boulder, New York, Toronto, Oxford, Lexington Books. HILDEBRAND David (ed.), (2014), Language or Experience, Special Issue of the European Journal of Pragmatism and American Philosophy, IV (2). Online: [journals.openedition.org/ejpap/275].

MOYAL-SHARROCK Daniele, (2016), “Can Wittgenstein be called a Pragmatist?,” Paradigmi, XXXIV (3), 67-85.

PERISSINOTTO Luigi, (2018), Introduzione a Wittgenstein, Bologna, il Mulino.

RORTY Richard, (1977), “Dewey's Metaphysics," in S. Cahn (ed.), New Studies in the Philosophy of John Dewey, Hanover, The University Press of New England, 45-74.

RORTY Richard, (2017 [1979]), La philosophie et le miroir de la nature, trad. T. Marchaisse, Paris, Éditions du Seuil.

RORTY Richard, (1982), “The World Well Lost,” Consequences of Pragmatism (Essays: 1972-1980), Minneapolis, The University of Minnesota Press.

STEINER Pierre, (2014), "Enacting Anti-Representationalism. The Scope and the Limits of Enactive Critiques of Representationalism," Avant, 5 (2), 43-86.

STEINER Pierre, (2017), "Pragmatism in Cognitive Science: from the Pragmatic Turn to Deweyan Adverbialism," Pragmatism Today, 8 (1), 9-27. 
STEINER Pierre, (2019), Désaturer l'esprit. Usages du pragmatisme, Paris, Questions Théoriques.

TIERCELIN Claudine, (2019), Pragmatism and Vagueness. The Venetian Lectures, Milano, Mimesis

International.

VARELA Francisco J., ROSCH Eleanor \& Evan THOMPSON, (1991), The Embodied Mind: Cognitive Science

and Human Experience, Cambridge-London, The MIT Press.

\section{NOTES}

1. Dans cet article, je ne traiterai pas du chapitre conclusif, qui expose une critique argumentée et largement partagée de la conception tendanciellement autonome de la méthode expérimentale de Dewey, ainsi qu'une problématisation de la technique en tant que question anthropologique et politique. Comme il s'agit d'une enquête importante mais collatérale à la trajectoire principale, je m'abstiendrai d'en parler ici.

2. À ce sujet, voir Hildebrand (2014).

3. Dewey parle de "sense," "feeling" et "significance" dans Experience and Nature (1981: 200), car il $\mathrm{y}$ a déjà des formes animales de signification, distinctes de la signification linguistique humaine ("meaning"). Les énactivistes parlent de "sense-making" en général, sans distinguer les différentes formes de signification, pour souligner la continuité du comportement culturel humain avec le comportement organique (Colombetti 2014: 16-7).

4. Mais il y a aussi des interprétations de la philosophie de Wittgenstein dans un sens naturaliste. Voir Moyal-Sharrock (2016).

\section{AUTEUR}

\section{ROBERTA DREON}

Università Ca' Foscari Venezia

robdre[at]unive.it 